\title{
Oncolytic cancer therapy with a vaccinia virus strain
}

\author{
LILI DENG ${ }^{1}$, JUN FAN $^{1}$, YUEDI DING ${ }^{1}$, JUE ZHANG $^{1}$, BIN ZHOU ${ }^{1}$, \\ YI ZHANG ${ }^{1}$, BIAO HUANG ${ }^{1}$ and ZHIGANG $\mathrm{HU}^{2}$ \\ ${ }^{1}$ Key Laboratory of Nuclear Medicine, Ministry of Health, Jiangsu Key Laboratory of Molecular Nuclear Medicine, \\ Jiangsu Institute of Nuclear Medicine, Wuxi, Jiangsu 214063; ${ }^{2}$ Clinical Laboratory, \\ Wuxi People's Hospital Affiliated to Nanjing Medical University, Wuxi, Jiangsu 214023, P.R. China
}

Received February 26, 2017; Accepted August 14, 2018

DOI: $10.3892 / o r .2018 .6801$

\begin{abstract}
Oncolytic vaccinia virus is currently undergoing evaluation as a biological anticancer agent in clinical trials. This treatment exploits the lytic nature of a viral infection to eradicate the tumor mass in a cancer cell-specific manner. So far, various vaccinia strains have been used as backbones in the design of oncolytic agents. However, the efficacy as oncolytic virotherapy of Chinese vaccinia strain Tian Tan (VTT) has not been reported. Vaccinia strain Guang9 (VG9), derived from VTT by consecutive plaque-cloning selection, was attenuated to a greater extent than its parental strain. In this study, the oncolytic efficacy of VG9 was evaluated. We examined in vitro replication and cytotoxicity, in vivo biodistribution, and antitumor effects in a B16 tumor model. The results revealed that VG9 replicated rapidly, but the cytotoxicity varied in different cell lines. Significant antitumor effects of VG9 were observed in a murine melanoma tumor model, and an antitumor cytotoxic T-lymphocyte response induced by VG9 was also observed. The results indicated that the Chinese vaccinia strain VG9 holds promise in the construction of a recombinant vaccinia virus vector and as a potential therapeutic strategy in cancer treatment.
\end{abstract}

\section{Introduction}

In recent years cancer has become one of the most serious threats to human health and life. Current cancer therapies, such as radiotherapy and chemotherapy, are less effective and

Correspondence to: Dr Biao Huang, Key Laboratory of Nuclear Medicine, Ministry of Health, Jiangsu Key Laboratory of Molecular Nuclear Medicine, Jiangsu Institute of Nuclear Medicine, 20 Qianrong Road, Wuxi, Jiangsu 214063, P.R. China

E-mail: huangbiao@jsinm.org

Dr Zhigang $\mathrm{Hu}$, Clinical Laboratory, Wuxi People's Hospital Affiliated to Nanjing Medical University, 299 Qingyang Road, Wuxi, Jiangsu 214023, P.R. China

E-mail: jswxhzg@163.com

Key words: oncolytic virus, vaccinia virus, Tian Tan strain Guang9, cancer therapy, virotherapy cause various side effects, therefore, novel strategies for cancer therapy are urgently needed. In the search for novel cancer therapies, oncolytic virotherapy has recently appeared as an appealing approach due to its ability to replicate in tumor cells with consequent spread to other cells (1-5), leading to significant oncolytic efficacy. In addition, oncolytic virotherapy can specifically kill through additional mechanisms such as arming therapeutic genes and causing tumor-specific cytotoxic T lymphocytes (CTL). Therefore, oncolytic virotherapy appears to be a promising approach to treat cancers that are refractory to current treatments.

At present, various viruses are used as replication-selective oncolytic viruses in the treatment of cancer, such as the adenovirus, herpes virus, Newcastle disease virus, and vaccinia virus (6-9). Among them, the vaccinia virus exhibits notable benefits such as intravenous stability, efficient delivery, large transgene-encoding capacity, verified ability to induce efficient immune responses, and a safe, live vaccine administered in humans. So far, a number of wild-type vaccinia strains have been used as backbones in the design of oncolytic agents such as Wyeth (10-16), Copenhagen (17) and Lister (18).

The vaccinia virus Tian Tan strain (VTT), the most widely used vaccine in China, played a critical role during the Chinese smallpox eradication campaign (19-21). The biological characteristics of VTT have already been studied systematically $(22,23)$. Briefly, VTT has a wide host cell range, and is less virulent than vaccinia virus Western Reserve strain (WR) but still remains neurovirulent. Some attenuated strains of VTT with lower toxicity were obtained by genetic modification (24-26). Of these, vaccinia virus strain Guang9 (VG9) displayed better attenuated properties as compared to its parental strain by using a traditional single plaque purification method (27-29). The neurovirulence and pathogenicity of VG9 were also notably lower (30), while the immunogenicity of VG9 was no less than that of VTT (31). Thus far, the biological characteristics of VG9 have been well studied and it is supposed to become an essential building block in the construction of a recombinant vaccinia virus vector. However, very few studies have evaluated the oncolytic efficacy of VG9, and no clinical application has been performed. In this study, we assessed the replication and cytotoxicity of VG9 in vitro, and evaluated the antitumor effects in a murine melanoma tumor model. Our findings will serve as a promising platform for further cancer therapy. 


\section{Materials and methods}

Cells and virus. Tumor cell lines including B16 (murine melanoma), Hepa 1-6 (murine hepatoma), HeLa (human cervix carcinoma), SGC-7901 (human gastric carcinoma), A549 (human lung carcinoma), MDA-MB-231 (human breast carcinoma) and normal cell line L-02 cells (human normal liver) were purchased from Shanghai Cell Collection (Shanghai, China). Vero (African green monkey kidney epithelial), BSC-40 (African green monkey kidney epithelial), and NIH3T3 (murine embryo fibroblast) cell lines were purchased from the American Type Culture Collection (ATCC; Manassas, VA, USA). All cells were cultured under the conditions suggested by the ATCC.

The vaccinia virus of Tian Tan strain VG9 was a gift from National Institutes for Food and Drug Control (NIFDC; Beijing, China). The titer of VG9 was determined by a plaque-forming assay on $\mathrm{BSC}-40$ cells.

In vitro viral replication. The replication ability of VG9 was observed in various cancer cell lines and normal cell lines at the multiplicity of infection (MOI) of $0.1 \mathrm{PFU} / \mathrm{cell}$. Cells pre-incubated in growth medium containing $2 \%$ fetal bovine serum (FBS) for $2 \mathrm{~h}$ were then washed and incubated in complete growth medium. Cells and supernatant were harvested at different time points (24, 48 and $72 \mathrm{~h}$ ), and viral titers were determined in BSC-40 cells after three cycles of freezing and thawing.

In vitro cytotoxicity assay. Cells (104/well) seeded in 96 well plates were infected with different MOIs of virus suspended in growth medium containing $2 \%$ FBS. Following cell culture at different time points $(24,48$ and $72 \mathrm{~h}), 20 \mu \mathrm{l}$ of $5 \mathrm{mg} / \mathrm{ml} \mathrm{3-(4,5-dimethyl-2-thiazolyl)-2,5-diphenyl-2-H-tet-}$ razolium bromide (MTT; Sigma-Aldrich; Merck KGaA, Darmstadt, Germany) was added to each well. Cells were incubated at $37^{\circ} \mathrm{C}$ for $4 \mathrm{~h}$, then the supernatants were discarded, and $150 \mu \mathrm{l}$ dimethylsulfoxide (DMSO) was added to each well and mixed thoroughly. After 10 mins of shaking, the color absorbance at $490 \mathrm{~nm}$ was measured by a spectrophotometric system (SpectraMax M5e; Molecular Devices, LLC, Sunnyvale, CA, USA).

Mice. The animal experiments were approved by the Institutional Animal Care and Use Committees (IACUC) of Jiangsu Institute of Nuclear Medicine (JSINM2010007). 20 female C57BL/6 immunocompetent mice (6 weeks old) were purchased from Shanghai Laboratory Animals Center (SLAC; Shanghai, China). They were housed under standard conditions (at $25^{\circ} \mathrm{C}$, with $40-50 \%$ humidity and a $12-\mathrm{h} / 12-\mathrm{h}$ light/dark cycle) and were given free access to diet and water.

In vivo viral replication. To evaluate in vivo viral replication, mice bearing subcutaneous B16 murine melanoma tumors were intraperitoneally injected with VG9 (1x10 7 PFU). After 5 days, brain, lung, liver, spleen, kidney and tumor tissue were harvested and homogenized. The viral yield was quantified by plaque assay on BSC-40 cells.

Tumor models and antitumor effects. To establish a murine melanoma tumor model, approximately $5 \times 10^{5}$ B16 cells in $100 \mu \mathrm{l}$ phosphate-buffered saline (PBS) were injected subcutaneously into the right flanks of C57BL/6 mice. PBS control), $10^{7}$ PFU of VG9 was injected intratumorally when tumors reached the size of 3-5 $\mathrm{mm}$ in diameter. Tumor growth was monitored every other day by computed tomography CT) scan. The tumor volume was calculated as the $\left[(\text { width })^{2} \mathrm{x}\right.$ length] $\mathrm{x} 0.52$ (32). Mice were euthanized when tumors reached their maximal permitted size according to the animal regulations, and Kaplan-Meier survival curves were plotted.

Measurement of neutralizing antibody to VG9. The titer of serum antibodies to virus was determined by time-resolved fluoroimmunoassay (TRFIA) (33). After coating 96-well plates with VG9 $(20 \mu \mathrm{g} / \mathrm{ml})$ overnight, diluted serum samples were incubated with the virus for $2 \mathrm{~h}$. After the plates were washed 6 times, $\mathrm{Eu}^{3+}$-labeled anti-mouse $\mathrm{IgG}$ secondary antibody (Cell Signaling Technology, Danvers, MA, USA) incubation followed for $1 \mathrm{~h}$. The fluorescent emission spectra of $\mathrm{Eu}^{3+}$ were obtained on a PerkinElmer LS-55 fluorescence spectrometer (PerkinElmer, Inc., Waltham, MA, USA) and time-resolved fluorescent measurements were carried out with an AutoDELFIA-1235 automatic analyzer (WALLAC; PerkinElmer, Inc.).

Cytotoxic T-lymphocyte study. After PBS or VG9-treatment for 13 days, spleens harvested from the PBS or virus-treated or from normal control mice were homogenized, filtered through a 40- $\mu$ m nylon strainer (BD Falcon; Becton Dickinson and Company, Franklin Lakes, NJ, USA) and cultured for $24 \mathrm{~h}$. B16 or Hepa 1-6 cells (1x10 4 cells/well) were cultured on 96-well plates and splenocytes were added at ratios of 10:1. Cell viability was measured by MTT assay after $48 \mathrm{~h}$.

Thyroid samples. Three surgically removed thyroid samples from 3 patients ( 1 male, 2 females, median age 52 years) were collected at the Department of Pathology of Jiangyuan Hospital Affiliated to Jiangsu Institute of Nuclear Medicine (Wuxi, China) in December of 2016. All patients provided informed consent before enrollment in the study, which was approved by the Ethics Committee of the Jiangyuan Hospital Affiliated to Jiangsu Institute of Nuclear Medicine.

Statistical analysis. Values are indicated as the mean \pm standard deviation (SD). Statistical analysis was calculated using the Mann-Whitney test for non-parametric data or Student's t-test for 2 independent samples when appropriate. Survival was calculated by Kaplan-Meier method, and differences between curves were assessed by log-rank test. All statistics were generated by SPSS 19.0 software (IBM Corp., Armonk, NY, USA).

\section{Results}

Replication of VG9 in vitro. The ability of VG9 to replicate and spread was determined in various cancer cell lines and two normal cell lines. The yield of infectious virus in cells at indicated time-points was quantified by plaque assays in BSC-40 cells. As shown in Fig. 1, VG9 rapidly increased in all cell types, reaching a maximum within $48 \mathrm{~h}$. The value either 
Table I. Cell viability of various cell lines infected with VG9 at different MOIs ${ }^{\mathrm{a}}$.

\begin{tabular}{|c|c|c|c|c|}
\hline \multirow[b]{2}{*}{ Cell lines } & \multicolumn{4}{|c|}{ Cell viability (\%) } \\
\hline & $0.01 \mathrm{MOI}$ & $0.1 \mathrm{MOI}$ & $1 \mathrm{MOI}$ & $10 \mathrm{MOI}$ \\
\hline B16 & $77.15 \pm 2.15$ & $37.50 \pm 2.47$ & $26.77 \pm 1.77$ & $12.30 \pm 1.18$ \\
\hline Hepa 1-6 & $59.73 \pm 1.41$ & $37.93 \pm 0.08$ & $33.47 \pm 1.05$ & $27.14 \pm 1.21$ \\
\hline A549 & $62.55 \pm 3.73$ & $53.32 \pm 1.69$ & $46.91 \pm 0.93$ & $40.40 \pm 1.90$ \\
\hline HeLa & $69.52 \pm 1.49$ & $52.55 \pm 0.69$ & $39.53 \pm 2.29$ & $26.08 \pm 3.09$ \\
\hline SGC-7901 & $70.35 \pm 4.22$ & $60.07 \pm 2.12$ & $29.75 \pm 1.59$ & $21.13 \pm 2.25$ \\
\hline MDA-MB-231 & $58.75 \pm 2.06$ & $48.61 \pm 1.33$ & $30.07 \pm 0.22$ & $15.24 \pm 2.70$ \\
\hline NIH3T3 & $104.04 \pm 5.75$ & $95.53 \pm 5.03$ & $80.41 \pm 2.26$ & $55.90 \pm 3.02$ \\
\hline L-02 & $96.60 \pm 1.96$ & $99.50 \pm 1.17$ & $95.25 \pm 2.93$ & $79.85 \pm 4.04$ \\
\hline
\end{tabular}

${ }^{\mathrm{a} F}$ Following infection for $72 \mathrm{~h}$, cell viability was assessed by MTT assay.

Table II. Cell viability of various cell lines infected with VG9 at different time-points ${ }^{\mathrm{a}}$.

\begin{tabular}{lrcr}
\hline & \multicolumn{3}{c}{ Cell viability $(\%)$} \\
\cline { 2 - 4 } Cell lines & \multicolumn{1}{c}{$24 \mathrm{~h}$} & $48 \mathrm{~h}$ & $72 \mathrm{~h}$ \\
\hline B16 & $77.33 \pm 3.06$ & $25.12 \pm 2.58$ & $12.30 \pm 1.18$ \\
Hepa 1-6 & $88.36 \pm 3.47$ & $38.06 \pm 1.29$ & $27.14 \pm 1.21$ \\
A549 & $86.67 \pm 2.09$ & $55.08 \pm 2.24$ & $40.40 \pm 1.90$ \\
HeLa & $80.36 \pm 2.11$ & $40.06 \pm 1.38$ & $26.08 \pm 3.09$ \\
SGC-7901 & $83.02 \pm 2.58$ & $38.13 \pm 3.26$ & $21.13 \pm 2.25$ \\
MDA-MB-231 & $72.06 \pm 3.48$ & $34.61 \pm 1.33$ & $15.24 \pm 2.70$ \\
NIH3T3 & $102.88 \pm 3.26$ & $80.41 \pm 2.66$ & $55.90 \pm 3.02$ \\
L-02 & $99.87 \pm 2.15$ & $82.96 \pm 3.23$ & $79.85 \pm 4.04$ \\
\hline
\end{tabular}

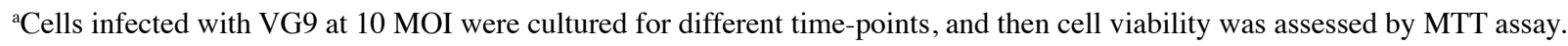

remained the same or changed slightly by $72 \mathrm{~h}$. Maximum virus production occurred in MDA-MB-231 cells, followed by B16 cells. VG9 titer in the two normal cells was lower as compared to that of the cancer cells. The results suggested natural tumor tropism of the vaccinia virus.

Cytotoxic effect in vitro. The oncolytic potency of VG9 was evaluated in various cell lines. Cells were cultured in 96-well plates and then infected with increasing doses of viruses. After 3 days infection, cell viability was assessed (Table I). The sensitivity to virus-induced cell killing varied between the cell lines. At an MOI of 1, >50\% of all cancer cells were killed. A viral MOI of 10 resulted in survival of $<20 \%$ in B16 cells or MDA-MB-231 cells; while $20-40 \%$ in other cell lines. Normal cells were poorly sensitive to virus-induced cell killing. Even when infected with an MOI of 10, 60-80\% of normal cells survived. The cell viability was also evaluated after infection at different time points (Table II). The results revealed that the cytotoxic effect of VG9 was time-dependent.

Replication of VG9 in vivo. The viral yield of VG9 in tumors and normal organ tissues was evaluated 5 days after infection.
Table III. Biodistribution of vaccinia viruses in tumor and normal tissues ${ }^{\mathrm{a}}$

\begin{tabular}{lc}
\hline Tissue & VG9 \\
\hline Tumor & $12.0(7.2-16) \times 10^{4}$ \\
Brain & $50(0-160)$ \\
Lung & $0(0-50)$ \\
Liver & $0(0-20)$ \\
Spleen & $80(16-240)$ \\
Kidney & $50(30-90)$ \\
\hline
\end{tabular}

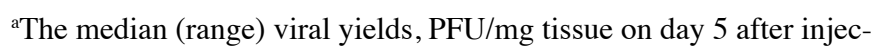
tion with VG9.

Harvested viruses were titered on BSC-40 cells and the yield was quantified per milligram of tissue. The results presented in Table III indicated that the viral yields of VG9 were significantly reduced in normal organs, while it was recovered at higher amounts in tumor tissue. 


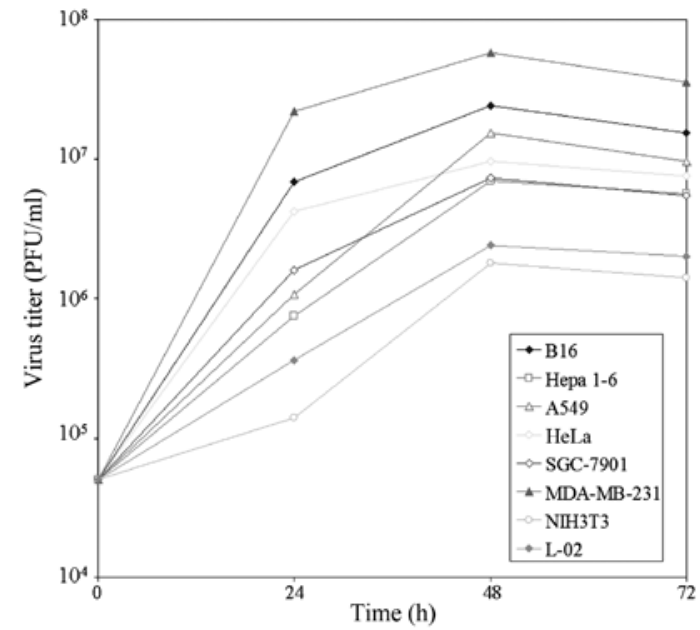

Figure 1. Viral replication of VG9 in vitro. Various cancer cell lines (B16, Hepa 1-6, A549, HeLa, SGC-7901 and MDA-MB-231) and two normal cell lines (NIH3T3 and L-02) were in fected with VG9 at $0.1 \mathrm{MOI}$ and samples were collected at indicated time-points. Virus titers were determined on BSC-40 cells.

Antitumor effect of VG9 in vivo. The ability of VG9 to function as an oncolytic virus was examined in a B16-murine melanoma tumor model. Immunocompetent C57BL/6 mice bearing subcutaneous B16 murine melanoma tumors were injected intratumorally with $1 \times 10^{7} \mathrm{PFU}$ of VG9 or PBS (control). Tumor development was monitored by CT (Fig. 2A). At 2 weeks from the initial treatment, tumors in the control group had significantly increased in size, while those in the VG9-treated groups had stabilized (Fig. 2B). All control mice died within 13 days, while VG9-treated mice lived longer with survival extended up to 28 days (Fig. $2 \mathrm{C}$ ).

Notably, the antitumor effect of VG9 was attributable to the replication of the virus alone as no therapeutic genes had been introduced into the virus. These results strongly indicated that VG9 had a notable antitumor effect as an oncolytic vaccinia virus.

Immune response induced by VG9. To evaluate the immune response against the virus itself, neutralizing antibody to virus was determined by time-resolved fluoroimmunoassay (TRFIA). As shown in Fig. 3A, neutralizing antibodies to VG9 were detectable by day 7 after injection and elevated through day 21. To assess the immune response against the target tumor, we evaluated tumor-specific CTL. Splenocytes harvested from VG9-treated or PBS-treated mice harboring B16 tumors or normal control mice were co-cultured with B16 or Hepa 1-6 cells. Cell viability assays revealed that VG9 induced a notable increase in B16-targeting CTL, while the effect was lost in Hepa 1-6 cells (Fig. 3B), indicating that vaccinia oncolysis induced tumor-specific immunity.

Oncolytic effect of VG9 on clinical samples. To further investigate the oncolytic effect of VG9 on clinical human tumor samples, we obtained three surgically resected human thyroid samples from Jiangyuan Hospital Affiliated to Jiangsu Institute of Nuclear Medicine and the oncolytic potency of VG9 was evaluated. Primary cells $\left(10^{4} /\right.$ well) from fresh thyroid tissue were cultured in 96-well plates. Three days
A
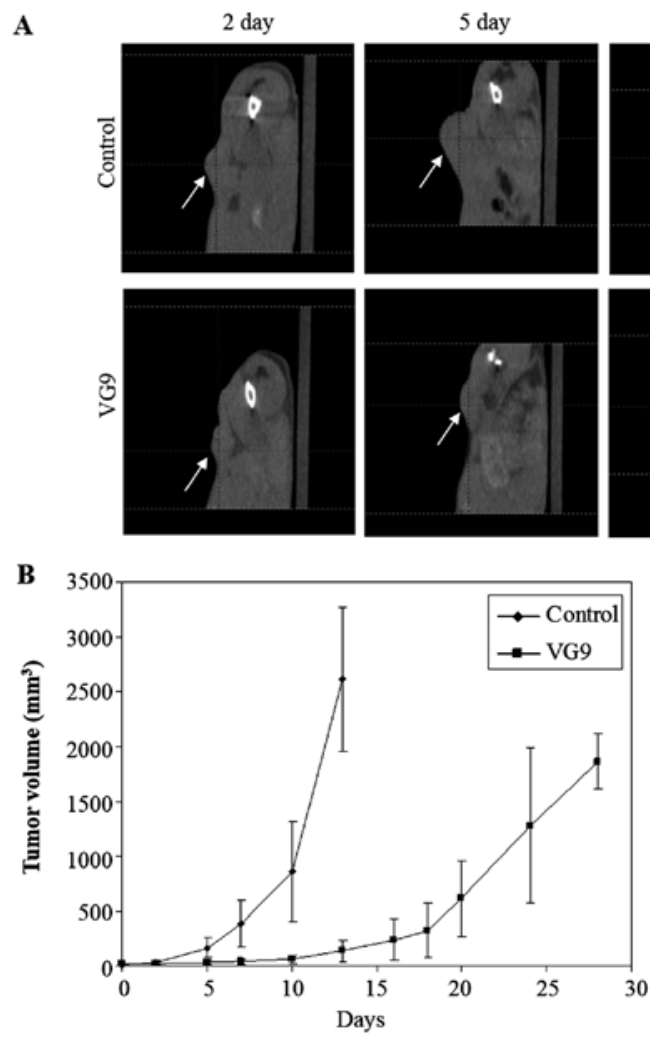

day

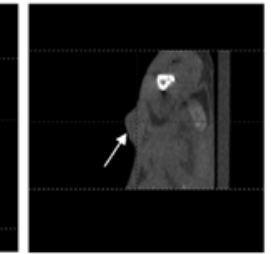

C

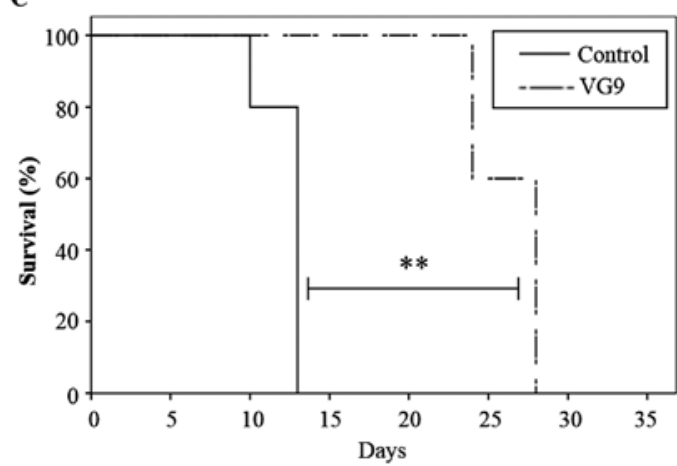

Figure 2. Antitumor efficacy of VG9 in a murine melanoma model. (A) Tumor development monitored by CT. (B) Mean tumor volume in mice treated with PBS (control group) and VG9 (VG9 group). (C) Kaplan-Meier survival curves for B16 tumor-bearing mice treated with PBS (control group) and VG9 (VG9 group) (** $\mathrm{P}<0.01$, Mantel-Cox test). $\mathrm{n}=5$ per group. 


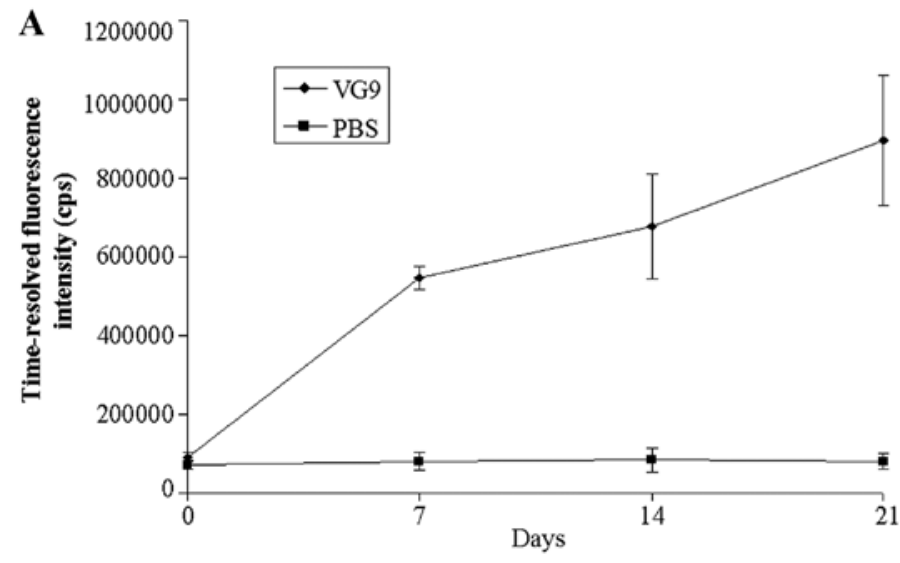

B

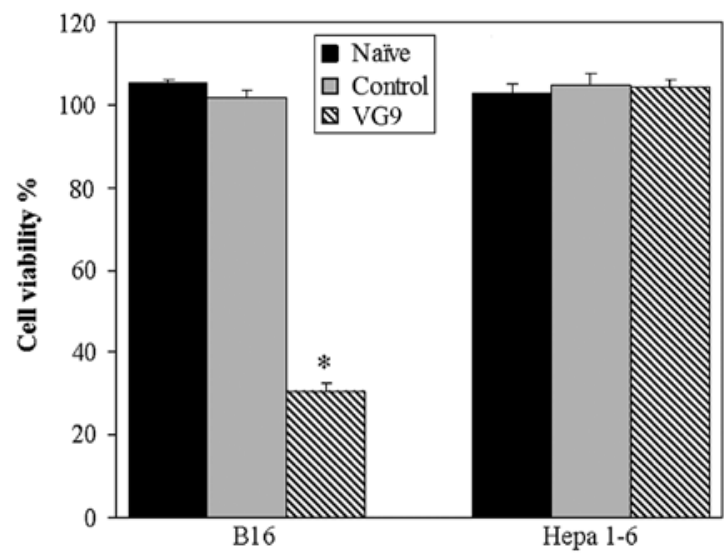

Figure 3. Immune response induced by VG9. (A) Anti-vaccinia neutralizing antibody development over time. (B) Cell viability of B16 and Hepa 1-6 cells cultured with splenocytes from naïve mice (naïve group) or murine melanoma tumor-bearing mice treated with PBS (control group) or VG9 (VG9 group). Each bar represents the mean $\pm \mathrm{SD}(\mathrm{n}=5) .{ }^{*} \mathrm{P}<0.05$ vs. the naïve or control group.

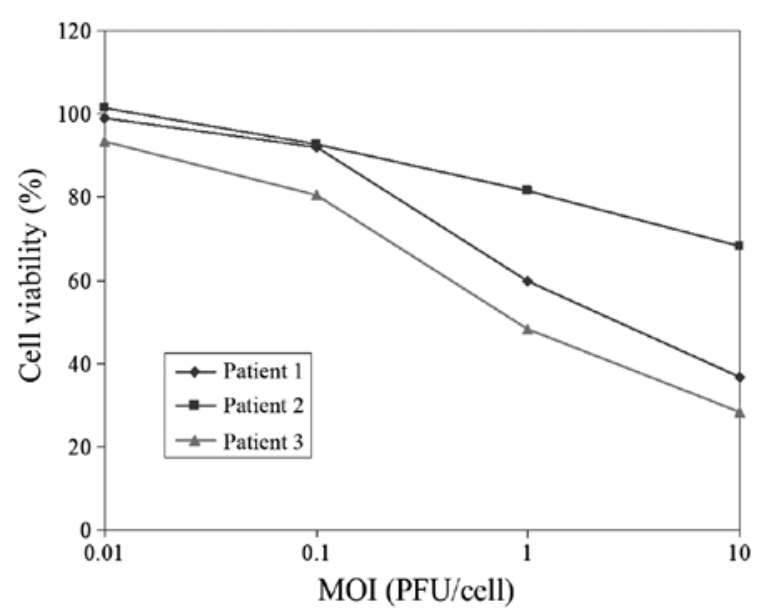

Figure 4. Oncolytic effect of VG9 on human thyroid tumor samples. Three surgically resected human thyroid samples from patients were harvested and primary cells $\left(10^{4} /\right.$ well) from fresh thyroid tissue were cultured in 96 -well plates. Three days after VG9 infection, cell viability was analyzed using the MTT cytotoxicity assay.

after VG9 infection, cell viability was analyzed using the MTT cytotoxicity assay (Fig. 4). The results revealed that VG9 induced a cytotoxic effect in patient 1 and 3 , while patient 2 was poorly sensitive to VG9-induced cell killing. A pathological test indicated that the thyroid samples from patients 1 and 3 were malignant while that of patient 2 was benign.

\section{Discussion}

We are interested in the research of cancer therapy using the vaccinia virus due to several favorable features. The lifecycle of vaccinia virus is short with mature virions forming within $6 \mathrm{~h}$ after infection (34), resulting in a high titer produced within a short period of time. The large transgene-encoding capacity of vaccinia virus facilitates multiple therapeutic strategies. Its native promoters are strong and efficient, leading to high levels of transgene expression using its own enzyme systems. There is a long history of the use of the vaccinia virus during the smallpox eradication and its biology is clear, making it safe and easy to use in humans. Notably, many laboratory studies and clinical trials have examined the applicability of several vaccine strains including Wyeth, Copenhagen and Lister. However, the potential of the Chinese vaccine strain as an oncolytic agent was previously untested. In this study, data characterizing the antitumor effect of Chinese vaccine virus Guang9 strain (VG9) in vitro and in vivo were presented. The results revealed that viral replication and cytotoxicity of VG9 was potent in vitro, and VG9 exhibited notable antitumor efficacy in inhibiting tumor development in a murine melanoma tumor model.

VG9 was derived from the Chinese vaccine Tian Tan strain (VTT) using consecutive plaque-cloning selection. According to research, VG9 produced a smaller necrosis area and pock diameter, less red swelling and lower incidences of fever and hyperpyrexia (27-29). Although VG9 still had neurotoxicity to a certain extent, the virulence was found to be lower 
than its parental virus (VTT) in various animal models (30). In previous studies, the neurovirulent vaccinia strain Western Reserve (WR), which has been widely used in laboratories and extensively tested in clinical trials, has an $\mathrm{LD}_{90}$ of $2.4 \mathrm{PFU}$, while VTT is about 5000-fold less virulent (23). Collectively, we conclude that VG9 may become an ideal vaccinia virus vector and a safer human vaccine. Some preliminary studies have indicated that removing the thymidine kinase gene of the vaccine virus may reduce the virulence as well as enhance tumor targeting $(35,36)$. Another approach to attenuate or enhance tumor-selective replication is the introduction of selected deletions in the viral genome (37-39). These constructions based on VG9 hold promise and the detailed oncolytic potency will be investigated in future studies. Our next step to improve VG9 will be to insert various therapeutic genes such as immune cytokine genes, suicide genes and enzyme-prodrug genes, to elevate its potency as well as maintain its high tumor selectivity.

Oncolytic viruses preferentially grow in tumor cells due to their natural tropism for cell surface proteins that are aberrantly expressed by tumor cells. In our in vitro study, the cytotoxic effect on tumor cells was much stronger, while normal cells were poorly sensitive to virally-induced cell killing. Our in vitro study also revealed the differences between the replication rates in different cancer cell lines. Vaccinia virus replicates in cytoplasm and needs a nucleotide pool for replication of the viral genome. Tumor cell lines have different pools of functional nucleotides, which produce different replication rates in various tumor cells. In addition, the growth rate of tumor cells is another factor. The ability of viral replication was evidently higher in fast-growing tumor cells, like highly malignant cells B16 and MDA-MB-231 cells. Another mechanism that may limit the overall effectiveness of oncolytic viruses is the susceptibility of cancer cells to apoptosis, which may be induced by viral infection or other factors. If cells undergo apoptosis too rapidly, this will reduce the time for viral replication and propagation.

The safety of the vaccinia virus is one of the most essential considerations for clinical applications. Since being used in smallpox vaccination programs globally, the safety of oncolytic vaccine viruses in humans has been demonstrated and specific antiviral agents are available $(40,41)$. Mild flu-like symptoms have been the primary side effects; no treatment-related changes in the parameters of hematological, hepatic, and renal function and no significant normal tissue toxicity has been reported to date $(10,12,42)$. In this study, there was no significant toxicity and no mice died even when $10^{9}$ PFU of VG9 was injected (data not shown). In some clinical studies, the dosage of the virus intravenous injection was $10^{8} \mathrm{PFU}$, while it was $10^{7} \mathrm{PFU}$ for intratumoral injection. Upon $10^{8} \mathrm{PFU}$ of VG9 treatment, similar results were observed with an insignificant change of the survival curve (data not shown). Furthermore, a higher concentration of the virus is not easy to disperse in tumors. Therefore, the dosage of $10^{7} \mathrm{PFU}$ was safe and enough. Due to its excellent safety in humans, novel cancer therapeutic strategies based on vaccinia backbones of the vaccinia virus are feasible to design, owing to its fast replication cycle and high selectivity for cancer tissue.

The rapid antiviral immune response and subsequent virus clearance, which limit the use of repeated injections, are potential limitations in the use of the vaccinia virus as an antitumor agent (43). To address this problem, one possible strategy is the administration of the vaccinia virus concurrently with tumor-trafficking immune cells, which would deliver viruses to their tumor targets (44). Another approach is using liposomes, polyethylene glycol, neutralizing antibodies, or other biological agents to disguise the vaccinia virus.

In this study, we revealed that the vaccinia strain VG9 alone, without therapeutic genes, can induce an antitumor effect by viral replication and consequent cell lysis. It has the potential to be a novel platform for cancer treatment with the ability to induce tumor destruction by multiple mechanisms and no cross-resistance with traditional therapies. However, hurdles such as the immune response, systemic distribution and intratumoral spread are major potential limitations and must be addressed in future studies.

\section{Acknowledgements}

We are grateful to the National Institutes for Food and Drug Control (NIFDC) for providing the vaccinia virus of the Tian Tan strain VG9.

\section{Funding}

The present study was supported by grants from the National Natural Science Foundation of China (no. 81703061).

\section{Availability of data and materials}

The datasets used during the present study are available from the corresponding author upon reasonable request.

\section{Authors' contributions}

LD and BH conceived the study. The manuscript was written by $L D$ and revised by $\mathrm{ZH}$. LD contributed to the viral replication. YZ carried out the cytotoxic assay. JF carried out the animal study and contributed to the design of the in vivo study. YD carried out the in vivo viral biodistribution. BZ contributed to the time-resolved fluoroimmunoassay and data acquisition. BH collected the clinical samples. $\mathrm{ZH}$ analyzed and interpreted the data. JZ contributed to analysis of data for the study. All authors read and approved the manuscript and agree to be accountable for all aspects of the research in ensuring that the accuracy or integrity of any part of the work are appropriately investigated and resolved.

\section{Ethics approval and consent to participate}

The animal experiments was approved by the Institutional Animal Care and Use Committees (IACUC) of Jiangsu Institute of Nuclear Medicine (JSINM2010007). The study was approved by the Ethics Committee of Jiangyuan Hospital Affiliated to Jiangsu Institute of Nuclear Medicine (Wuxi, China). All patients provided informed consent before enrollment in the study.

\section{Patient consent for publication}

Not applicable.

\section{Competing interests}

The authors declare that they have no competing interests. 


\section{References}

1. Kirn D, Martuza RL and Zwiebel J: Replication-selective virotherapy for cancer: Biological principles, risk management and future directions. Nat Med 7: 781-787, 2001.

2. Everts B and van der Poel HG: Replication-selective oncolytic viruses in the treatment of cancer. Cancer Gene Ther 12: 141-161, 2005 .

3. Thorne SH, Hermiston T and Kirn D: Oncolytic virotherapy: Approaches to tumor targeting and enhancing antitumor effects Semin Oncol 32: 537-548, 2005.

4. Liu TC, Galanis E and Kirn D: Clinical trial results with oncolytic virotherapy: A century of promise, a decade of progress. Nat Clin Pract Oncol 4: 101-117, 2007.

5. Guo ZS and Bartlett DL: Oncolytic viruses as platform for multimodal cancer therapeutics: A promising land. Cancer Gene Ther 21: 261-263, 2014

6. Heise C, Sampson-Johannes A, Williams A, McCormick F Von Hoff DD and Kirn DH: ONYX-015, an E1B gene-attenuated adenovirus, causes tumor-specific cytolysis and antitumoral efficacy that can be augmented by standard chemotherapeutic agents. Nat Med 3: 639-645, 1997.

7. Walker JR, McGeagh KG, Sundaresan P, Jorgensen TJ, Rabkin SD and Martuza RL: Local and systemic therapy of human prostate adenocarcinoma with the conditionally replicating herpes simplex virus vector G207. Hum Gene Ther 10: 2237-2243, 1999

8. Phuangsab A, Lorence RM, Reichard KW, Peeples ME and Walter RJ: Newcastle disease virus therapy of human tumor xenografts: Antitumor effects of local or systemic administration. Cancer Lett 172: 27-36, 2001.

9. Puhlmann M, Gnant M, Brown CK, Alexander HR and Bartlett DL: Thymidine kinase-deleted vaccinia virus expressing purine nucleoside phosphorylase as a vector for tumor-directed gene therapy. Hum Gene Ther 10: 649-657, 1999.

10. Mastrangelo MJ, Maguire HC Jr, Eisenlohr LC, Laughlin CE, Monken CE, McCue PA, Kovatich AJ and Lattime EC: Intratumoral recombinant GM-CSF-encoding virus as gene therapy in patients with cutaneous melanoma. Cancer Gene Ther 6: 409-422, 1999.

11. Kim JH, Oh JY, Park BH, Lee DE, Kim JS, Park HE, Roh MS, Je JE, Yoon JH, Thorne SH, et al: Systemic armed oncolytic and immunologic therapy for cancer with JX-594, a targeted poxvirus expressing GM-CSF. Mol Ther 14: 361-370, 2006.

12. Park BH, Hwang T, Liu TC, Sze DY, Kim JS, Kwon HC, Oh SY, Han SY, Yoon JH, Hong SH, et al: Use of a targeted oncolytic poxvirus, JX-594, in patients with refractory primary or metastatic liver cancer: A phase I trial. Lancet Oncol 9: 533-542, 2008

13. Hwang TH, Moon A, Burke J, Ribas A, Stephenson J, Breitbach CJ, Daneshmand M, De Silva N, Parato K, Diallo JS et al: A mechanistic proof-of-concept clinical trial with JX-594, a targeted multi-mechanistic oncolytic poxvirus, in patients with metastatic melanoma. Mol Ther 19: 1913-1922, 2011.

14. Heo J, Reid T, Ruo L, Breitbach CJ, Rose S, Bloomston M, Cho M, Lim HY, Chung HC, Kim CW, et al: Randomized dose-finding clinical trial of oncolytic immunotherapeutic vaccinia JX-594 in liver cancer. Nat Med 19: 329-336, 2013.

15. Thorne SH, Hwang TH, O'Gorman WE, Bartlett DL, Sei S, Kanji F, Brown C, Werier J, Cho JH, Lee DE, et al: Rational strain selection and engineering creates a broad-spectrum, systemically effective oncolytic poxvirus, JX-963. J Clin Invest 117: 3350-3358, 2007.

16. Kirn DH, Wang Y, Le Boeuf F, Bell J and Thorne SH: Targeting of interferon-beta to produce a specific, multi-mechanistic oncolytic vaccinia virus. PLoS Med 4: e353, 2007.

17. Foloppe J, Kintz J, Futin N, Findeli A, Cordier P, Schlesinger Y, Hoffmann C, Tosch C, Balloul JM and Erbs P: Targeted delivery of a suicide gene to human colorectal tumors by a conditionally replicating vaccinia virus. Gene Ther 15: 1361-1371, 2008.

18. Zhang Q, Yu YA, Wang E, Chen N, Danner RL, Munson PJ, Marincola FM and Szalay AA: Eradication of solid human breast tumors in nude mice with an intravenously injected light-emitting oncolytic vaccinia virus. Cancer Res 67: 10038-10046, 2007.

19. Hui X and Yutu J: The eradication of smallpox in Shanghai, China, October 1950-July 1951. Bull World Health Organ 59: 913-917, 1981.

20. Ma B: Variolation, pioneer of modern immunology. Zhonghua Yi Shi Za Zhi 25: 139-144, 1995 (In Chinese).

21. Henderson DA: The eradication of smallpox - an overview of the past, present, and future. Vaccine 29 (Suppl 4): D7-D9, 2011.
22. Jin Q, Chen L, Chen S, Huang J, Feng Z, Yuan J, Jin D, Bai H and Hou Y: Characterization of the complete genomic sequence of the vaccinia virus Tian Tan strain. Sci China 27: 562-567, 1997.

23. Fang Q, Yang L, Zhu W, Liu L, Wang H, Yu W, Xiao G, Tien P, Zhang $L$ and Chen $Z$ : Host range, growth property, and virulence of the smallpox vaccine: Vaccinia virus Tian Tan strain. Virology 335: 242-251, 2005.

24. Huang X, Lu B, Yu W, Fang Q, Liu L, Zhuang K, Shen T, Wang H, Tian P, Zhang L, et al: A novel replication-competent vaccinia vector MVTT is superior to MVA for inducing high levels of neutralizing antibody via mucosal vaccination. PLoS One 4: e4180, 2009.

25. Liu Z, Wang S, Zhang Q, Tian M, Hou J, Wang R, Liu C, Ji X, Liu Y and Shao Y: Deletion of C7L and K1L genes leads to significantly decreased virulence of recombinant vaccinia virus TianTan. PLoS One 8: e68115, 2013.

26. Yu W, Fang Q, Zhu W, Wang H, Tien P, Zhang L and Chen Z: One time intranasal vaccination with a modified vaccinia Tiantan strain MVTT(ZCI) protects animals against pathogenic viral challenge. Vaccine 28: 2088-2096, 2010.

27. Products NiftCoPaB: A summary report on the selection of VG9 vaccinia virus strain which acquired in the collaborative work of the national smallpox vaccine. Compil Commun Vac Virus Strains (Beijing): 45-49, 1974.

28. Products CIoB: Comparison of reactogenicity in experimental animals of five vaccinia virus strains of domestic and abroad. 7: 70-73, 1978.

29. Products CIoB: Comparison of reactogenicity and immunogenicity in experimental animals of five vaccinia virus strains of domestic and abroad. 4: 13-19, 1978.

30. Zhu R, Huang W, Wen Z, Wang W, Zhou Y and YC. W: Studies on the virulence of a novel attenuated vaccinia virus VG9 strain in animals. Zhongguo Bingdubing Zazhi 1: 183-187, 2011.

31. Zhu R, Huang W, Wang Y and Yu Y: Immunogenicity of an attenuated vaccinia virus VG9strain.Zhongguo Shengwuzhipinxue Zazhi 9: 347-350, 2011

32. O'Reilly MS, Boehm T, Shing Y, Fukai N, Vasios G, Lane WS, Flynn E, Birkhead JR, Olsen BR and Folkman J: Endostatin: An endogenous inhibitor of angiogenesis and tumor growth. Cell 88: 277-285, 1997.

33. Huang B, Tao W, Shi J, Tang Land Jin J: Determination of ochratoxin A by polyclonal antibodies based sensitive time-resolved fluoroimmunoassay. Arch Toxicol 80: 481-485, 2006

34. Zeh HJ and Bartlett DL: Development of a replication-selective, oncolytic poxvirus for the treatment of human cancers. Cancer Gene Ther 9: 1001-1012, 2002.

35. Buller RM, Smith GL, Cremer K, Notkins AL and Moss B: Decreased virulence of recombinant vaccinia virus expression vectors is associated with a thymidine kinase-negative phenotype. Nature 317: 813-815, 1985.

36. Gnant MF, Puhlmann M, Bartlett DL and Alexander HR Jr: Regional versus systemic delivery of recombinant vaccinia virus as suicide gene therapy for murine liver metastases. Ann Surg 230: 352-361, 1999.

37. Ramsey-Ewing A and Moss B: Restriction of vaccinia virus replication in CHO cells occurs at the stage of viral intermediate protein synthesis. Virology 206: 984-993, 1995.

38. Kan S, Wang Y, Sun L, Jia P, Qi Y, Su J, Liu L, Yang G, Liu L, Wang Z, et al: Attenuation of vaccinia Tian Tan strain by removal of viral TC7L-TK2L and TA35R genes. PLoS One 7: e31979, 2012.

39. Guo ZS, Naik A, OMalley ME, Popovic P, Demarco R, Hu Y, Yin X, Yang S, Zeh HJ, Moss B, et al: The enhanced tumor selectivity of an oncolytic vaccinia lacking the host range and antiapoptosis genes SPI-1 and SPI-2. Cancer Res 65: 9991-9998, 2005.

40. De Clercq E: Cidofovir in the therapy and short-term prophylaxis of poxvirus infections. Trends Pharmacol Sci 23: 456-458, 2002.

41. Wittek R: Vaccinia immune globulin: Current policies, preparedness, and product safety and efficacy. Int J Infect Dis 10: 193-201, 2006

42. Breitbach CJ, Burke J, Jonker D, Stephenson J, Haas AR, Chow LQ, Nieva J, Hwang TH, Moon A, Patt R, et al: Intravenous delivery of a multi-mechanistic cancer-targeted oncolytic poxvirus in humans. Nature 477: 99-102, 2011

43. Evgin L, Acuna SA, Tanese de Souza C, Marguerie M,Lemay CG, Ilkow CS, Findlay CS, Falls T, Parato KA, Hanwell D, et al: Complement inhibition prevents oncolytic vaccinia virus neutralization in immune humans and cynomolgus macaques. Mol Ther 23: 1066-1076, 2015.

44. Thorne SH, Liang W, Sampath P, Schmidt T, Sikorski R, Beilhack $\mathrm{A}$ and Contag $\mathrm{CH}$ : Targeting localized immune suppression within the tumor through repeat cycles of immune cell-oncolytic virus combination therapy. Mol Ther 18: 1698-1705, 2010 